\title{
Compositions inside a rectangle and unimodality
}

\author{
Bruce E. Sagan
}

Received: 9 July 2007 / Accepted: 6 May 2008 / Published online: 24 May 2008

(C) Springer Science+Business Media, LLC 2008

\begin{abstract}
Let $c^{k, l}(n)$ be the number of compositions (ordered partitions) of the integer $n$ whose Ferrers diagram fits inside a $k \times l$ rectangle. The purpose of this note is to give a simple, algebraic proof of a conjecture of Vatter that the sequence $c^{k, l}(0), c^{k, l}(1), \ldots, c^{k, l}(k l)$ is unimodal. The problem of giving a combinatorial proof of this fact is discussed, but is still open.
\end{abstract}

Keywords Composition · Integer partition · Unimodal

\section{Introduction}

Let $\mathbb{N}$ and $\mathbb{P}$ denote the nonnegative and positive integers, respectively. A partition of $n \in \mathbb{N}$ is a weakly decreasing sequence $\lambda=\left(\lambda_{1}, \ldots, \lambda_{r}\right)$ of positive integers called parts such that $\sum_{i} \lambda_{i}=n$. We write $\lambda \vdash n$ or $|\lambda|=n$ if $\lambda$ partitions $n$. We will also use the notation $\lambda=\left(n^{m_{n}}, \ldots, 1^{m_{1}}\right)$ where $m_{i}$ is the number of times $i$ appears as a part in $\lambda$. If $m_{i}=1$ then the exponent is suppressed and if $m_{i}=0$ then so is the base. The Ferrers diagram of $\lambda$, also denoted $\lambda$, consists of left-justified rows of squares with $\lambda_{i}$ squares in row $i$. The Ferrers diagram of $\lambda=(4,3,3,1)=\left(4,3^{2}, 1\right)$ is shown in Figure 1.

Partitions can be ordered by letting $\lambda \leq \mu$ if the Ferrers diagram for $\lambda$ is contained in the upper left corner of the one for $\mu$. Equivalently, $\lambda_{i} \leq \mu_{i}$ for all $i$ where we set $\lambda_{i}=0$ if $i$ is greater than the number of parts of $\lambda$ and similarly for $\mu$. The set of partitions under this partial order is called Young's lattice. More information about partitions and this lattice can be found in the books of Andrews [2], Sagan [12], or Stanley [18].

B.E. Sagan $(\bowtie)$

Department of Mathematics, Michigan State University, East Lansing, MI 48824-1027, USA

e-mail: sagan@math.msu.edu 

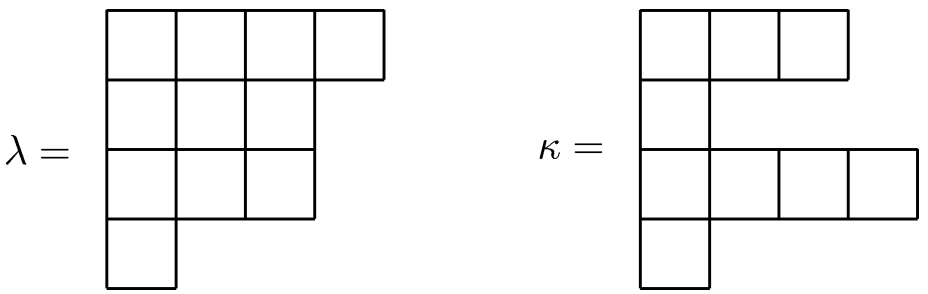

Fig. 1 Ferrers diagrams for the partition $\lambda=(4,3,3,1)$ and the composition $\kappa=(3,1,4,1)$

We say that $\lambda$ fits inside a $k \times l$ rectangle if $\lambda \leq\left(l^{k}\right)$. In other words, $\lambda$ has at most $k$ parts each of size at most $l$. Let $p^{k, l}(n)$ denote the number of such $\lambda$ where $\lambda \vdash n$. A sequence $a_{0}, a_{1}, \ldots, a_{r}$ of nonnegative integers is said to be unimodal if there is an index $m$ such that

$$
a_{0} \leq a_{1} \leq \ldots \leq a_{m} \geq a_{m+1} \geq \ldots \geq a_{r} .
$$

Unimodal sequences arise in many aspects of combinatorics, geometry, and algebra. See the survey articles of Stanley [16] and Brenti [4] for details. Our interest is in the following well-known theorem.

Theorem 1.1 Given $k, l \in \mathbb{P}$ the sequence

$$
p^{k, l}(0), p^{k, l}(1), \ldots, p^{k, l}(k l)
$$

is unimodal.

This result was first proved by Sylvester [20] using invariant theory. Since then, there have been a number of other proofs. In particular, Stanley [15] derived this and much more from the Hard Lefschetz Theorem of algebraic geometry. Proctor [11] was able to reduce Stanley's proof to pure linear algebra. And finally, Kathy O'Hara [10] gave a combinatorial proof of this theorem.

A composition $\kappa$ of $n$, written $\kappa \models n$, is any sequence $\kappa=\left(\kappa_{1}, \ldots, \kappa_{r}\right)$ of positive integers summing to $n$. Note that a composition need not be weakly decreasing. All of the definitions discussed so far have obvious analogues for compositions so we will not bother restating them. For example, the Ferrers diagram of the composition $(3,1,4,1)$ is displayed in Figure 1. Although there is a large literature surrounding partitions, compositions have only recently aroused interest due to their connection with quasi-symmetric functions [5, 6], the theory of patterns [7, 14], and the subword and factor partial orders $[3,9,13]$.

Let $c^{k, l}(n)$ be the number of compositions of $n$ fitting inside a $k \times l$ rectangle. In this note we will give a simple, algebraic proof of the following conjecture of Vatter [personal communication].

Theorem 1.2 Given $k, l \in \mathbb{P}$ the sequence

$$
c^{k, l}(0), c^{k, l}(1), \ldots, c^{k, l}(k l)
$$

is unimodal. 
We should note that Andrews [1] proved the analogous result if one considers compositions with exactly $k$ parts. One can also consider the case where one has $k$ parts and zero is allowed as a part. But this is seen to be equivalent to what Andrews did by adding 1 to each part.

In the next section, we will prove this result by passing to the generating function of the sequence. The final section will include some comments and an indication about how a combinatorial proof of Vatter's conjecture might go.

\section{Unimodality of the composition sequence}

Let $a_{0}, a_{1}, \ldots, a_{r}$ be a sequence of real numbers and let $q$ be a variable. We consider the corresponding generating function $f(q)=a_{0}+a_{1} q+\cdots+a_{r} q^{r}$. By convention, we let $a_{i}=0$ if $i<0$ or $i>r$. We will say that $f(q)$ has a given property if the sequence itself does.

We will need the standard $q$-analogue of $n$, namely

$$
[n]=1+q+q^{2}+\cdots+q^{n-1} .
$$

It is well known that the generating function for the sequence $p^{k, l}(n), 0 \leq n \leq k l$, is the $q$-binomial coefficient

$$
\left[\begin{array}{c}
k+l \\
l
\end{array}\right]=\frac{[k+l] !}{[k] ![l] !}
$$

where $[k] !=[k][k-1] \cdots[1]$. So a restatement of Theorem 1.1 is that the $q$-binomial coefficients are unimodal.

To prove the analogous result about compositions, we will need a result about products of polynomials. It is not true, in general, that the product of two unimodal polynomials is unimodal. For example, if $f(q)=1+q+q^{2}+2.3 q^{3}+2 q^{4}$ then

$$
f(q)^{2}=1+2 q+3 q^{2}+6.6 q^{3}+9.6 q^{4}+8.6 q^{5}+9.29 q^{6}+9.2 q^{7}+4 q^{8} .
$$

But there is a situation in which unimodality is preserved. A sequence $a_{0}, a_{1}, \ldots, a_{r}$ is log concave if $a_{i}^{2} \geq a_{i-1} a_{i+1}$ for all $i$ with $0<i<r$. Keilson and Gerber [8] gave the following nice characterization of $\log$ concavity.

Proposition 2.1 (Keilson and Gerber) Let $f(q)$ be a polynomial with positive coefficients. Then $f(q)$ is log concave if and only if $f(q) g(q)$ is unimodal for any unimodal polynomial $g(q)$ with positive coefficients.

Now let

$$
f^{k, l}=f^{k, l}(q)=\sum_{n \geq 0} c^{k, l}(n) q^{n} .
$$

Our main result is as follows.

Theorem 2.2 Let $k, l \in \mathbb{P}$. 
(a) If $k \geq 2$ then

$$
f^{k, l}=1+q[l] f^{k-1, l} .
$$

(b) We have

$$
f^{k, l}=\sum_{i=0}^{k}(q[l])^{i} .
$$

(c) The polynomial $f^{k, l}$ is unimodal.

Proof. (a) Let $K^{k, l}$ be the set of compositions fitting inside a $k \times l$ rectangle, and let $K^{k, l}\langle r\rangle \subseteq K^{k, l}$ be those compositions with first part equal to $r$. So we have the disjoint union

$$
K^{k, l}=\{\epsilon\} \uplus\left(\biguplus_{r=1}^{l} K^{k, l}\langle r\rangle\right)
$$

where $\epsilon$ denotes the empty composition. Removing the first part of any $\kappa \in K^{k, l}$ leaves a composition in $K^{k-1, l}$. So translating the union above into a generating function gives the desired result.

(b) This is an easy induction on $k$ using the recursion in part (a).

(c) We again induct on $k$. Clearly $f^{1, l}=[l+1]$, so we are done in the base case. If $k \geq 2$ then using part (a) and the previous proposition finishes the proof.

\section{Comments and open questions}

\subsection{Log concavity and symmetry}

Another relationship between unimodality and log concavity is the following wellknown result.

Proposition 3.1 Let $a_{0}, a_{1}, \ldots, a_{r}$ be a sequence of positive real numbers. If the sequence is log concave, then it is also unimodal.

Sometimes to prove a sequence is unimodal, it is actually easier to prove that it satisfies the stronger log-concavity condition. This is because proving unimodality directly may involve finding the index where the sequence is maximized, and that can be highly nontrivial. However, the sequence $p^{k, l}(n), 0 \leq n \leq k l$, is not log concave in general. So it should come as no surprise that neither is $c^{k, l}(n), 0 \leq n \leq k l$, and for much the same reason. In particular, if $k, l \geq 2$ then both sequences start $1,1,2$ which already violates log concavity.

Another common property of sequences is symmetry. Say that $a_{0}, a_{1}, \ldots, a_{r}$ is symmetric if $a_{i}=a_{r-i}$ for all $i, 0 \leq i \leq r$. By taking complements in the rectangle, 
it is easy to see that $p^{k, l}(n), 0 \leq n \leq k l$, is symmetric. In general, this property is not shared by compositions in a rectangle. For example, if $k=l=2$ then the corresponding sequence is $1,1,2,2,1$.

\subsection{Lower order ideals}

Let $(P, \leq)$ be a poset (partially ordered set). Definitions for terms from the theory of posets which are not given here can be found in Stanley's book [17, Chapter 3]. A lower order ideal is $L \subseteq P$ such that $x \in L$ and $y \leq x$ implies $y \in L$. The principal lower order ideal generated by $x$ is the order ideal

$$
L(x)=\{y \in P \mid y \leq x\} .
$$

Let $Y$ and $K$ denote Young's lattice and the poset of all compositions, respectively. Then the set of partitions in a rectangle is the order ideal $Y\left(l^{k}\right)$ and similarly for compositions.

If $x, y \in P$ then $x$ is covered by $y$, written $x \lessdot y$, if $x<y$ and there is no $z$ with $x<z<y$. An $x-y$ chain of length $n$ in $P$ is a subposet of the form $x=x_{0}<x_{1}<$ $\ldots<x_{n}=y$. This chain is saturated if each inequality is actually a cover. A poset is graded if it has a unique minimal element denoted $\hat{0}$, a unique maximal element denoted $\hat{1}$, and every saturated $\hat{0}-\hat{1}$ chain has the same length. If $P$ is graded and $x \in P$ then all saturated $\hat{0}-x$ chains have the same length, called the rank of $x$ and denoted $\mathrm{rk} x$. In this case, the $n$th rank of $P$ is the subposet

$$
P_{n}=\{x \in P \mid \operatorname{rk} x=n\} .
$$

We will say that a graded poset $P$ has a property if the sequence of cardinalities

$$
\left|P_{0}\right|,\left|P_{1}\right|, \ldots,\left|P_{r}\right|
$$

has that property, where $r=\mathrm{rk} \hat{1}$. We will sometimes preface the property by "rank-" if clarification is needed. So Theorem 1.1 can be restated as saying that the poset $Y\left(l^{k}\right)$ is unimodal. It is natural to ask whether $Y(\lambda)$ is unimodal for all partitions $\lambda$. But this is too much to ask for, as demonstrated by the following theorem of Stanton [19].

Theorem 3.2 (Stanton) The lower order ideal $Y(8,8,4,4)$ is not unimodal.

In view of Stanton's result, it is perhaps surprising that all principal lower order ideals in the composition poset $K$ are unimodal. Given a graded poset $P$, we let $f^{P}=f^{P}(q)$ be the generating polynomial for the sequence (3). The proof of the following theorem is so much like that of Theorem 2.2 that we omit it.

Theorem 3.3 Consider a composition $\kappa \in K$.

(a) Suppose $\kappa=\left(\kappa_{1}, \ldots, \kappa_{s}\right) \neq \epsilon$, and let $l=\kappa_{1}$ and $\gamma=\left(\kappa_{2}, \ldots, \kappa_{s}\right)$. Then

$$
f^{K(\kappa)}=1+q[l] f^{K(\gamma)} .
$$

(b) The polynomial $f^{K(\kappa)}$ is unimodal. 


\subsection{A combinatorial proof?}

Theorem 2.2 is so easy to prove algebraically, one would think that there is also an easy combinatorial proof. But so far one has not been found. Here we present a possible approach in the hopes that someone may be able to push it through.

Let $P$ be poset. A chain decomposition of $P$ is a family of saturated chains $C_{1}, \ldots, C_{a}$ such that $P=\uplus_{i} C_{i}$. If $P$ is graded then we say an $x-y$ chain in $P$ is symmetric if rk $y=\operatorname{rk} \hat{1}-\operatorname{rk} x$. A symmetric chain decomposition or $S C D$ is a chain decomposition where all the chains are symmetric. It is easy to see that if $P$ has a SCD then its rank sequence is symmetric and unimodal.

O'Hara [10] constructed her ground-breaking combinatorial proof of Theorem 1.1 as follows. Let $Z(\lambda)$ be the poset of all partitions in $Y(\lambda)$ ordered by $\mu<v$ if and only if $|\mu|<|\nu|$. So for any partition $\lambda, Z(\lambda)$ has the same set of ranks as does $Y(\lambda)$, but many more covering relations in general.

Theorem 3.4 (O'Hara) Given $k, l \in \mathbb{P}$, the poset $Z\left(l^{k}\right)$ has a SCD.

We note that it is still an open problem to give a SCD for $Y\left(l^{k}\right)$.

As mentioned above, $K\left(l^{k}\right)$ is not always rank-symmetric. But we can replace symmetry by another condition. If $P$ is graded then we say that a chain decomposition is modal (a MCD) if there is some rank $P_{m}$ such that every $C_{i}$ contains an element of $P_{m}$. We call $P_{m}$ a modal rank. Note that a MCD may have more than one modal rank, but all modal ranks have the same number of elements.

It is not hard to see that if a poset $P$ has a MCD then it is rank-unimodal. It would be very interesting to find a MCD for $K\left(l^{k}\right)$ or even for some related poset that has the same ranks. Note that a modal rank $P_{m}$ for $P=K\left(l^{k}\right)$ seems to occur when

$$
m=\lceil k(l+1) / 2\rceil-1
$$

where $\lceil\cdot\rceil$ is the ceiling function. Also note that there are other partial orders on the set of compositions $[3,9,13]$ and they have the same set of ranks as $K\left(l^{k}\right)$. Of these, the partial order we are considering has the fewest covers. So in may be useful to consider one of the other orders instead.

Acknowledgement I would like to thank Adam Goyt and Vince Vatter for interesting discussions.

\section{References}

1. Andrews, G.E.: A theorem on reciprocal polynomials with applications to permutations and compositions. Amer. Math. Monthly 82(8), 830-833 (1975)

2. Andrews, G.E.: The theory of partitions. Cambridge Mathematical Library. Cambridge University Press, Cambridge (1998). Reprint of the 1976 original

3. Björner, A., Sagan, B.E.: Rationality of the Möbius function of a composition poset. Theoret. Comput. Sci. 359(1-3), 282-298 (2006)

4. Brenti, F.: Log-concave and unimodal sequences in algebra, combinatorics, and geometry: an update. In: Jerusalem combinatorics '93. Contemp. Math., vol. 178, pp. 71-89. Amer. Math. Soc., Providence (1994)

5. Ehrenborg, R.: On posets and Hopf algebras. Adv. Math. 119(1), 1-25 (1996) 
6. Gessel, I.M.: Multipartite $P$-partitions and inner products of skew Schur functions. In: Combinatorics and algebra, Boulder, Colo., 1983. Contemp. Math., vol. 34, pp. 289-317. Amer. Math. Soc., Providence (1984)

7. Heubach, S., Mansour, T.: Avoiding patterns of length three in compositions and multiset permutations. Adv. Appl. Math. 36(2), 156-174 (2006)

8. Keilson, J., Gerber, H.: Some results for discrete unimodality. J. Amer. Statist. Assoc. 66, 386-389 (1971)

9. Kitaev, S., Liese, J., Remmel, J., Sagan, B.E.: Rationality, irrationality, and Wilf equivalence in generalized factor order (2008, in preparation)

10. O'Hara, K.M.: Unimodality of Gaussian coefficients: a constructive proof. J. Combin. Theory Ser. A 53(1), 29-52 (1990)

11. Proctor, R.A.: Solution of two difficult combinatorial problems with linear algebra. Amer. Math. Monthly 89(10), 721-734 (1982)

12. Sagan, B.E.: The symmetric group: Representations, combinatorial algorithms, and symmetric functions, 2nd edn. Graduate Texts in Mathematics, vol. 49. Springer, New York (2001).

13. Sagan, B.E., Vatter, V.: The Möbius function of a composition poset. J. Algebraic Combin. 24(2), 117-136 (2006)

14. Savage, C.D., Wilf, H.S.: Pattern avoidance in compositions and multiset permutations. Adv. in Appl. Math. 36(2), 194-201 (2006)

15. Stanley, R.P.: Weyl groups, the hard Lefschetz theorem, and the Sperner property. SIAM J. Algebraic Discrete Methods 1(2), 168-184 (1980)

16. Stanley, R.P.: Log-concave and unimodal sequences in algebra, combinatorics, and geometry. In: Graph theory and its applications: East and West, Jinan, 1986. Ann. New York Acad. Sci., vol. 576, pp. 500-535. New York Acad. Sci., New York (1989)

17. Stanley, R.P.: Enumerative Combinatorics, vol. 1. Cambridge Studies in Advanced Mathematics, vol. 49. Cambridge University Press, Cambridge (1997). With a foreword by Gian-Carlo Rota, Corrected reprint of the 1986 original

18. Stanley, R.P.: Enumerative Combinatorics, vol. 2. Cambridge Studies in Advanced Mathematics, vol. 62. Cambridge University Press, Cambridge (1999). With a foreword by Gian-Carlo Rota and appendix 1 by Sergey Fomin

19. Stanton, D.: Unimodality and Young's lattice. J. Combin. Theory Ser. A 54(1), 41-53 (1990)

20. Sylvester, J.J.: Proof of the hitherto undemonstrated fundamental theorem of invariants. In: The collected mathematical papers of James Joseph Sylvester, vol. 3, pp. 117-126. Cambridge University Press, Cambridge (1973) 